\title{
Changes in behavior as side effects in methylphenidate treatment: review of the literature
}

This article was published in the following Dove Press journal:

Neuropsychiatric Disease and Treatment

12 October 2016

Number of times this article has been viewed

\author{
Doris Susanne Konrad- \\ Bindl' \\ Ursula Gresser' \\ Barbara Maria Richartz ${ }^{2}$ \\ 'Department of Internal Medicine, \\ University of Munich, Munich, \\ ${ }^{2}$ Clinic der Jägerwinkel, Bad Wiessee, \\ Germany
}

Background: Our review of the scientific literature focused on an analysis of studies describing instances of methylphenidate treatment leading (or not) to behavioral changes in the pediatric, adolescent, and adult populations.

Materials and methods: We conducted a literature search in PubMed, Medline, and Google using the keywords "methylphenidate", "behavioral changes", "adverse effects", and "side effects". A total of 44 studies were identified as reporting on the effects and adverse effects of methylphenidate administration, and were included in the analysis.

Results: Five studies specifically set out to study, record, and discuss changes in behavior. Eight studies did not set out to study behavioral effects, but record and discuss them. A total of 28 studies recorded behavioral effects, but failed to discuss these further. Three studies did not include behavioral effects.

Conclusion: This review records what data have been published in respect of changes in behavior in association with the use of methylphenidate. While there is some evidence to suggest that methylphenidate causes changes in behavior, the majority of the studies reviewed paid little or no attention to this issue. Based on the available data, it is impossible to determine the point at which such behavioral effects occur. The frequency of occurrence of behavioral effects is also impossible to determine with certainty. Based on the available data, it is not possible to rule out whether behavioral effects may persist or not persist once treatment is discontinued. In conclusion, despite countless publications and extensive administration, especially to children, we have insufficient data to judge the long-term effects and risks of methylphenidate taking. Keywords: methylphenidate, changes in behavior, attention-deficit/hyperactivity disorder, children, adults, adverse effects

\section{Introduction}

Methylphenidate is used primarily in the treatment of children, adolescents, and adults with attention-deficit/hyperactivity disorder (ADHD). Furthermore, it is used in the treatment of narcolepsy, major depression disorder, and in patients with terminal cancer. Methylphenidate is usually prescribed for oral or transdermal use. As methylphenidate increases the ability to concentrate, it is sometimes used to enhance cognitive ability. In the treatment of ADHD, the aim of methylphenidate is to help to improve ADHD symptoms, such as difficulty paying attention or being hyperactive or impulsive. In 2012, Britton stated that until 2011, not a single study had been published on the long-term effects of treatment with methylphenidate. ${ }^{1}$ Since then, preclinical research has identified possible changes in brain structure and function, which suggest that "early pharmacotherapy can produce neuroadaptations that can persist long after drug treatment has ended".
Outpatient Clinic for Internal Medicine, University of Munich, 5 Blombergstrasse, Sauerlach, Munich 82054, Germany

Tel +498104887822

Fax +498104887824

Email ursulagresser@email.de (c) (i) () 2016 Konrad-Bindl et al. This work is published and licensed by Dove Medical Press Limited. The full terms of this license are available at https://www.dovepress.com/terms.php (c) ${ }_{\mathrm{BY}} \mathrm{NC}$ and incorporate the Creative Commons Attribution - Non Commercial (unported, v3.0) License (http://creativecommons.org/licenses/by-nc/3.0/). By accessing the work you hereby accept the Terms. Non-commercial uses of the work are permitted without any further permission from Dove Medical Press Limited, provided the work is properly attributed. For permission for commercial use of this work, please see paragraphs 4.2 and 5 of our Terms (https://www.dovepress.com/terms.php). 
In rats, exposure to methylphenidate during preadolescence has been shown to lead to behavioral changes, such as increased depressive symptoms, as well as a reduction in the ability to adapt to a familiar environment. ${ }^{2}$ Adult rats treated with methylphenidate during adolescence were shown to be less responsive to positive stimuli (such as a novel environment or sex) and more susceptible to stressful situations. ${ }^{3}$ Bolaños et al stated that:

[our] findings show that developmental administration of MPH [methylphenidate] results in aberrant behavioral adaptations during adulthood, and these results underscore the need for further developmental research geared toward a better understanding of the mechanisms underlying MPHinduced behavioral plasticity. ${ }^{3}$

In a study of seven healthy adult humans, a dose of $0.25 \mathrm{mg} / \mathrm{kg}$ body weight or higher resulted in the inhibition of more than $50 \%$ of dopamine transporters. ${ }^{4}$ Long-term treatment with methylphenidate in humans led to significantly increased activity in the caudate and putamen regions. Wang et al suggested that the increased activity of dopamine transporters compensates for their pharmacological inhibition, resulting in increased inattention and the need for an increase in the dose administered. ${ }^{5}$ There are a plethora of studies investigating changes in brain structure and function, but the focus in this publication was on clinical studies.

In her review of studies investigating the neuropsychological effects of methylphenidate, Advokat concluded that the use of stimulant drugs, such as methylphenidate or amphetamine, leads to increased attention and concentration, but does not result in improved learning or academic achievements. The author explained that her review of more than 30 years of research involving children, adolescents, and students failed to show evidence of the desired effects in any of these age-groups. The author expressed concerns regarding the administration of stimulant drugs, such as methylphenidate, as these may lead to reduced impulsiveness, which in turn could result in impaired performance in cognitive tasks requiring flexibility and adaptability. ${ }^{6}$

In November 2015, a Cochrane review was published with the aim of assessing the beneficial and harmful effects of methylphenidate for children and adolescents with ADHD. The authors stated:

At the moment, the quality of the available evidence means that we cannot say for sure whether taking methylphenidate will improve the lives of children and adolescents with ADHD. ${ }^{7}$
Changes in behavior can be defined as gradual yet permanent changes affecting the defining features of an individual's personality without causing significant impairment in intellectual ability. ${ }^{8}$ For this study, we included the following symptoms as changes in behavior: abnormal behavior, accidental injury, agitation, aggression, anxiety, depression, depressed mood, disinterested in others, dizziness, drowsiness, emotional lability, euphoria, feeling jittery, irritability, mood alteration, nail biting, nervousness, nightmares, prone to crying, psychomotor hyperactivity, restlessness, sadness, staring, talks less, and tics. Methylphenidate can cause cardiovascular, abdominal, psychiatric, growth and development, and neurological side effects. For this study, the focus was on psychiatric symptoms. Methylphenidate targets the central nervous system by inhibiting the reuptake of dopamine. ${ }^{4,8-11}$ To a lesser extent, it also inhibits the reuptake of norepinephrine, ${ }^{9-11}$ and binds to the serotonin transporter, albeit with low affinity. $9,11,12$

Chemically speaking, methylphenidate belongs to the amphetamines. ${ }^{9}$ In 1981, it was included as part of the licensed prescription drugs listed in annex III of the newly amended German Narcotic Drugs Act (Betäubungsmittelgesetz). Amendments to the professional guidelines on the prescription of stimulant drugs (Arzneimittel-Richtline) made by the Federal Ministry of Health in 2010 meant that only "specialists in behavioral disorders of children and/or adolescents" would be allowed to prescribe stimulant drugs for use in the treatment of ADHD. ${ }^{13}$ Our review of the scientific literature focused on an analysis of studies describing instances of methylphenidate treatment leading (or not) to behavioral changes.

\section{Materials and methods}

We conducted a literature search in PubMed, Medline, and Google using the keywords "methylphenidate", "behavioral changes", "adverse effects", and "side effects". A total of 44 studies were identified as reporting on the effects and adverse effects of methylphenidate administration, and were included in the analysis. In some studies, the results were divided among several publications. The publication period for articles was from 1990 until 2015. We did our last search on July 9, 2015. We included studies focusing on methylphenidate. We included studies with more medicines than methylphenidate if they were focusing on methylphenidate. We excluded studies with more medicines than methylphenidate if they were focusing on medicines other than methylphenidate. We excluded studies focusing only on physiological side effects like changes in blood pressure. 
Long-term effects of the drug were one of the main issues under consideration during the selection of studies. However, the current body of literature contains only a small number of articles on the long-term effects of methylphenidate. There was significant variation among the 44 studies reviewed, some of which were blind, others open-label. Some of the comparison groups received a placebo, while others received a different drug product. Some studies compared different forms of methylphenidate, while others compared methylphenidate at different doses.

Comparison was rendered more difficult by some of the studies involving small numbers of participants and being of limited duration: $57 \%$ of the studies lasted for less than 80 days, and patients in the shortest study ${ }^{14}$ received the drug for only 5 days. Despite its small size, Silva et al ${ }^{14}$ was judged to be of good quality, due to its randomized, double-blind, placebo-controlled, crossover trial design, and was included in the review.

Three of the studies had fewer than 30 participants. Bron et al, ${ }^{15}$ which involved 27 adult participants, was selected due to its good-quality study design, and the fact that participants had not previously been exposed to methylphenidate. Ghuman et $\mathrm{al}^{16}$ was selected due to the fact that it involved 27 very young participants (aged 3-5 years), which meant that methylphenidate was used off-label. All of the participants were methylphenidate-naïve, and treatment duration was relatively long compared to the other studies, lasting between 3 and 24 months. The study did not receive any funding, and in contrast to the other studies reviewed, did not include participants with concomitant psychiatric disorders. The smallest study selected was Maayan et al, ${ }^{17}$ which involved nine very young participants, all of whom were aged between 4 and 5 years and methylphenidate-naïve.

Some studies were included in the review despite the fact that they did not focus on side effects. One such example was Barbaresi et al, ${ }^{18}$ a retrospective cohort study, which followed participants from birth until the age of 17.2 years. While the authors described the long-term treatment with methylphenidate as effective, their conclusion was not based on the use of specific assessment tools. Assessment was by means of responses from participants and teachers, who were asked to rate the effect of treatment (positive effect, no effect, or not known). While the authors used their data to argue that long-term treatment with stimulant drugs had a positive effect, their data should be regarded as of questionable quality. Not only is it impossible to check whether the medication was administered at all, but questions regarding the effectiveness of treatment were too generic, and both students and teachers may have been overly positive in their responses.

Smith et al, ${ }^{19}$ who failed to provide any information on side effects, included results from as early as 1989. A longitudinal study conducted over 14 years, it is nonetheless worthy of mention, not only due to its sheer length but also because it showed no difference between participants receiving the drug and a comparison group from the general population. Analysis and discussion of results were modeled on Aagaard and Hansen. ${ }^{20}$ While the article by Aagaard and Hansen also included data on amphetamine, atomoxetine, and modafinil, we did not include any studies focusing on these medicines. The review by Aagaard and Hansen was published in 2011. From this paper, we included 14 studies in our review: Biederman et al, ${ }^{21}$ Arabgol et al, ${ }^{22}$ Maayan et al, ${ }^{17}$ Amiri et al, ${ }^{23}$ Findling et al, ${ }^{24}$ Newcorn et al, ${ }^{25}$ Greenhill et al, ${ }^{26}$ Gau et al, ${ }^{27}$ Silva et al, ${ }^{14}$ Kemner et al, ${ }^{28}$ Swanson et al, ${ }^{29}$ and Kratochvil et al. ${ }^{30}$ We identified and included an additional 29 studies: eleven of these included adult participants only, or participants between 4 and 65 years of age. Another ten, which involved children and adolescents, were published between 2011 and 2015. We also included an additional nine studies that had been published in 2010 or earlier. An overview of the studies is listed in Table 1.

Table I Overview of all 44 studies

\begin{tabular}{|c|c|c|c|c|c|c|}
\hline Study & $\begin{array}{l}\text { Treatment } \\
\text { period }\end{array}$ & Follow-up & $\begin{array}{l}\text { Patients } \\
\text { completed (n) }\end{array}$ & Age (years) & $\begin{array}{l}\text { Focus on } \\
\text { side effects }\end{array}$ & Withdrawal (\%) \\
\hline Adler et $\mathrm{al}^{51}$ & $\begin{array}{l}5 \text { weeks }+ \\
6 \text { months }\end{array}$ & None & $\begin{array}{l}218 \text { (5 weeks), } \\
\text { I70 (6 months) }\end{array}$ & $18-60$ & Yes & 19.7 \\
\hline Adler et $\mathrm{al}^{31}$ & $6-12$ months & None & 550 & $18-65$ & Yes & 18.5 \\
\hline Bejerot et $\mathrm{al}^{52}$ & 2 years & None & 133 & $31.1 \pm 10.9$ & No & 17 \\
\hline Bron et $\mathrm{al}^{15}$ & 6 weeks & None & 27 & $18-55$ & No & II.I \\
\hline Buitelaar et $\mathrm{al}^{32}$ & 12 weeks & None & 401,370 (OL) & $18-63$ & Yes & $4.3,5(\mathrm{OL})$ \\
\hline Buitelaar et $\mathrm{al}^{65}$ & 56 weeks & None & 155 (OL), 45 (Wd) & $18-65$ & Yes & $10.4(\mathrm{OL})$ \\
\hline Casas et $\mathrm{al}^{33}$ & 14 weeks & I week & 279 & $18-65$ & No & I6.9 (GI), $20.7(G 2)$, I (P) \\
\hline Fredriksen et $\mathrm{al}^{53}$ & 12 months & None & 250 & $18-60$ & No & 12 \\
\hline
\end{tabular}

(Continued) 
Table I (Continued)

\begin{tabular}{|c|c|c|c|c|c|c|}
\hline Study & $\begin{array}{l}\text { Treatment } \\
\text { period }\end{array}$ & Follow-up & $\begin{array}{l}\text { Patients } \\
\text { completed (n) }\end{array}$ & Age (years) & $\begin{array}{l}\text { Focus on } \\
\text { side effects }\end{array}$ & Withdrawal (\%) \\
\hline Huss et $\mathrm{al}^{54,55}$ & 66 weeks & None & 725 & $18-60$ & Yes & II.3 (PI), 3.8 (P2), 5 (P3), 2.7 (ES) \\
\hline Kooij et al ${ }^{45}$ & 7 weeks & None & 45 & $20-56$ & No & 0 \\
\hline Valdizán-Usón et al ${ }^{62}$ & I year & None & 730 & $4-65$ & No & NK \\
\hline Amiri et $\mathrm{al}^{23}$ & 6 weeks & None & 60 & $6-15$ & No & 0 \\
\hline Arabgol et $\mathrm{al}^{22}$ & 6 weeks & None & 33 & $6.5-16$ & No & 6.23 \\
\hline Barbaresi et al ${ }^{18}$ & 33.8 months & 17.2 years & 283 & 9.8 mean age & No & NK \\
\hline Barkley et $\mathrm{al}^{46}$ & 30 days & None & 83 & $5-13$ & Yes & 3.6 \\
\hline Biederman et $\mathrm{al}^{21}$ & 19 weeks & None & 161 & $6-14$ & No & 4.35 \\
\hline Cherland and Fitzpatrick ${ }^{63}$ & 21 months & None & 98 & $4-17$ & Yes & NK \\
\hline Döpfner et al ${ }^{56}$ & $6-12$ weeks & None & 822 & $7-16$ & No & 3.16 \\
\hline Efron et al ${ }^{47}$ & 4 weeks & None & 125 & $\begin{array}{l}\text { I04.8 months, } \\
\text { mean age }\end{array}$ & Yes & 3.2 \\
\hline Findling et $\mathrm{al}^{24}$ & 3 weeks & None & 318 & $6-12$ & No & 6.6 \\
\hline Garg et $\mathrm{al}^{57}$ & 8 weeks & None & 69 (33 МPH) & $6-14$ & Yes & 18.18 \\
\hline Gau et $\mathrm{a}^{27}$ & 28 days & None & 64 & $6-15$ & No & 0 \\
\hline Ghuman et $\mathrm{al}^{16}$ & 24 months & None & 27 & $3-5$ & No & 6 \\
\hline Greenhill et $\mathrm{al}^{26}$ & 9 weeks & None & 97 & $6-17$ & No & 0 \\
\hline Haertling et $\mathrm{al}^{58}$ & 12 weeks & None & 262 & $10.9 \pm 2.5$ & No & NK \\
\hline Karabekiroglu et al ${ }^{59}$ & I 5 days & None & 90 & $5-16$ & Yes & 8.9 \\
\hline Kemner et $\mathrm{al}^{28}$ & 21 days & None & I,323 (850 MPH) & $6-12$ & No & 4.8 \\
\hline Khajehpiri et $\mathrm{al}^{60}$ & 6 months & None & 71 & $4-15$ & Yes & 0 \\
\hline Kratochvil et $\mathrm{al}^{30}$ & 10 weeks & None & 228 (44 MPH) & $\begin{array}{l}\text { 7-15 months, } \\
7-9 \text { weeks }\end{array}$ & No & I I.4 \\
\hline Lee et $\mathrm{al}^{48}$ & 2 weeks & None & 157 & $6-12$ & Yes & NK \\
\hline Maayan et al ${ }^{17}$ & 4 weeks & None & II & $4-5$ & No & 27 \\
\hline MTA group ${ }^{34-39}$ & I4 months & $\begin{array}{l}24 \text { months, } \\
6 \text { and } 8 \text { years }\end{array}$ & 579 & $7-9.9$ & No & $<1$ \\
\hline Newcorn et $\mathrm{al}^{25}$ & 12 weeks & 6 weeks & 516 & $6-16$ & No & 5 \\
\hline PATS group ${ }^{40-42}$ & 70 weeks & $\begin{array}{l}3,4 \text {, and } \\
6 \text { years }\end{array}$ & 165 & $3-5.5$ & Yes & II \\
\hline Pelham et $\mathrm{al}^{49}$ & 3 weeks & None & 70 & $6-12$ & No & 0 \\
\hline Smith et al ${ }^{19}$ & 14 years & Longitudinal & $|3|$ & $1-17$ & No & NK \\
\hline Ramtvedt et al ${ }^{64}$ & 6 weeks & None & 34 & I I.3 mean age & Yes & 2.94 \\
\hline Robb et $a^{61}$ & 6-8 weeks & None & 45 & $6-12$ & Yes & 13.3 \\
\hline Silva et al $^{14}$ & 2 weeks & None & 54 & $6-12$ & No & I (P) \\
\hline Steele et $\mathrm{al}^{66}$ & 8 weeks & None & 145 & $6-12$ & No & 11.6 \\
\hline Stein et $\mathrm{al}^{50}$ & 4 weeks & None & 47 & $5-16$ & Yes & NK \\
\hline Swanson et $\mathrm{al}^{29}$ & 21 days & None & 184 & $6-12$ & No & 0 \\
\hline Wigal et $\mathrm{al}^{44}$ & 12 weeks & 30 days & 230 & $6-18$ & No & 2 \\
\hline Zarinara et $\mathrm{al}^{43}$ & 6 weeks & None & 38 & $6-13$ & No & 5.3 \\
\hline
\end{tabular}

Abbreviations: ES, extension study; GI, group I; MPH, methylphenidate; NK, not known; SE, side effect; OL, open-label; PI, Phase I; P, placebo; Wd, withdrawal.

\section{Results}

\section{Study design}

Studies included in the analysis differed in terms of study design, and included randomized parallel-group studies with a placebo arm (13), ${ }^{21-26,31-44}$ randomized crossover studies (ten),,$^{14,15,29,36,45-50}$ open-label studies (17),,$^{17,19,27,28,30,32,51-61}$ and retrospective studies (four). ${ }^{16,18,62,63}$

\section{Inclusion criteria}

Thirteen of the studies required participants to be methylphenidate-naïve. ${ }^{15-17,19,23,40-42,44,45,47,53,59,60,64}$ In 19 of the studies, methylphenidate was discontinued between 1 and
30 days prior to the start of the study. $14,22,24,25,28-33,44,48-51,61,65,66$ Seven of the studies did not specify whether participants had had previous exposure to methylphenidate. ${ }^{18,27,34-39,46,52,57,62,63}$ Six of the studies included both methylphenidate-naïve participants and participants with prior exposure to methylphenidate. . $^{21,26,54-56,58}$

\section{Funding arrangements}

Nineteen of the studies were funded by pharmaceutical companies. ${ }^{18,21,24,25,27-29,31-33,44,49,51,54,56,58,61,65,66}$ Ten were funded using government or university funding. ${ }^{19,23,34-39,43,45,47,48,50,52,64}$ Four of the studies declared only conflict of interest, ${ }^{15,17,26,40-42,64}$ 
Five were not in receipt of funding, ${ }^{16,22,53,57,62}$ while six failed to provide information on funding arrangements. ${ }^{14,30,46,59,60,63}$

\section{Comparison group}

In 21 of the studies, the comparison group received a placebo. ${ }^{14,15,21,24-26,29,32,33,40-42,44-46,48-51,54,61,64,65}$ One of the studies compared methylphenidate with modafinil, ${ }^{23}$ four of the studies compared it with atomoxetine, ${ }^{25,28,30,57}$ one with reboxetine, ${ }^{22}$ three with dexamphetamine, ${ }^{47,52,64}$ and one with venlafaxine. ${ }^{43}$ Five of the studies compared different forms of methylphenidate, ${ }^{24,27,29,49,66}$ while four compared methylphenidate at different doses. ${ }^{33,40-42,46,50}$ Ten of the studies did not include a comparison group. ${ }^{16-18,31,53,56,58-60,62}$

\section{Study duration}

Seven of the studies were conducted over a period of between 11 and 21 days, ${ }^{14,24,28,29,48,49,59}$ five lasted for between 22 and 40 days, ${ }^{17,27,46,47,50} 12$ lasted for between 41 and 80 days, ${ }^{15,22,23,26,30,43,45,56,57,61,64,66}$ and 20 for more than 80 days. ${ }^{16,18,19,21,25,31-42,44,51-54,58,60,62,63,65}$

\section{Study population}

In ten of the studies, participants were adults aged between 18 and 65 years. . $^{15,31-33,45,51-55,65}$ Of the 2,783 participants included in these studies, 1,498 were males. In 33 of the studies, participants were aged between 3 and 17 years. Of the 6,620 included in these studies, 3,678 were males. Three of the studies did not provide any information on sex distribution among participants. ${ }^{34-39,48,63}$ In one of the studies, 590 of the 730 participants aged between 4 and 65 years were males. $^{62}$

\section{Size of studies}

Three of the studies had fewer than 30 participants, ${ }^{15-17}$

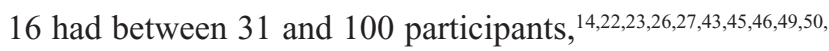
57,59,60,61,63,64 18 between 101 and 500 participants, , $^{18,19,21,24,29,30}$ $32,33,40-42,44,47,48,51-53,58,65,66$ while seven had more than 500 participants. $^{25,28,31,34-39,54,56,62}$

\section{Rates of withdrawal due to side effects}

Among the studies reviewed, withdrawal rates due to side effects were found to be between 0 and $27 \%$. Eight of the studies had a zero withdrawal rate $14,23,26,27,29,45,49,60$ (one of these recorded an early withdrawal by one participant allocated to the placebo group). ${ }^{14}$ Seven of the studies did not provide information on either the number of withdrawals or withdrawal rate due to side effects. ${ }^{18,19,48,50,58,62,63}$

\section{Classification of side effects}

Of the studies, 27 did not provide information on the methods or definitions employed in the reporting of side effects. In one of the studies, side effects were reported if experienced by at least $1 \%$ of participants, ${ }^{28}$ in four studies the cutoff was at least $2 \%$ of participants, ${ }^{26,29,32,49}$ in one study at least $3 \%$ of participants, ${ }^{24}$ in seven studies at least $5 \%,{ }^{25,30,33,44,54,55,61,65}$ and in two studies at least $10 \% .^{31,66}$ Two of the studies recorded the most commonly observed side effects only. ${ }^{51,40-42}$

\section{Frequency of side effects}

Frequency of side effects varied enormously between studies. In Haertling et al, $13.7 \%$ of participants reported experiencing side effects. ${ }^{58}$ In Khajehpiri et al, the figure was $100 \% .^{60}$ Sixteen studies failed to provide this information. ${ }^{17,19,23,27,29,30,40-44,47,49,50,59,63}$

\section{Behavioral changes}

Side effects associated with changes in behavior are listed in Table 2. Five studies specifically addressed the issue of behavioral changes, and duly recorded and described these. ${ }^{40-42,51,59,63,64}$ Cherland and Fitzpatrick focused on the psychotic side effects of methylphenidate. ${ }^{63}$ The other studies addressed behavioral changes in relation to the long-term safety of methylphenidate, ${ }^{51}$ its short-term side effects, ${ }^{63}$ its safety and tolerability, ${ }^{40-42}$ and its use in minimizing adverse events. ${ }^{64}$

The most frequently recorded symptoms were "irritability" (0\%-80\%), "prone to crying" (1\%-71\%), "staring" (21\%-62\%), "anxiety" (2\%-61\%), "sadness" (0\%-56\%), "nail biting" (22\%-45\%), "euphoria" (16\%-43\%), and "talks less" (27\%-42\%). Among studies with adult participants, the most commonly recorded symptoms were "drowsiness" $(0 \%-32 \%)$ and "nervousness" (3\%-23\%).

Table 2 does not include individual cases of severe side effects. Casas et $\mathrm{al}^{33}$ and Huss et $\mathrm{al}^{54,55}$ mentioned one attempted suicide each. Fredriksen et $\mathrm{al}^{53}$ explained that the suicidal thoughts reported by one of the participants in their study were classed as not drug-induced, as they occurred several months after methylphenidate had been discontinued. Adler et $\mathrm{al}^{31}$ listed side effects reported by more than $10 \%$ of participants; $1.8 \%$ of their participants were reported as experiencing psychiatric symptoms, such as mania, aggression, and paranoia. Bejerot et $\mathrm{al}^{52}$ reported that three of their participants with concomitant psychiatric disorders developed such symptoms as hallucinations, agitation, and aggression, or seizures. Kemner et $\mathrm{al}^{28}$ reported that participants treated with methylphenidate developed severe side effects, such as mania, paranoia, crying/fear/aggression, and kicking the table. Symptoms ceased once treatment was discontinued. 
Table 2 Overview of behavioral changes observed in persons treated with methylphenidate

\begin{tabular}{|c|c|c|c|c|c|c|c|c|c|c|c|c|c|}
\hline Study & \multicolumn{2}{|l|}{$\begin{array}{l}\text { Limit for } \\
\text { symptoms (\%) }\end{array}$} & $\begin{array}{l}\text { Abnormal } \\
\text { behavior }\end{array}$ & \multicolumn{2}{|c|}{$\begin{array}{l}\text { Accidental } \\
\text { injury }\end{array}$} & Agitation & \multicolumn{2}{|c|}{ Aggression } & \multicolumn{2}{|c|}{ Anxiety } & \multicolumn{2}{|c|}{ Depression } & $\begin{array}{l}\text { Depressed } \\
\text { mood }\end{array}$ \\
\hline Adler et $\mathrm{a}^{51}$ & $* 1)$ & & - & - & & $5 \%$ & - & & $12 \%$ & & - & & - \\
\hline Adler et $\mathrm{a}^{31}$ & 10 & & - & - & & - & - & & $13 \%$ & & - & & - \\
\hline Bejerot et a ${ }^{52}$ & - & & - & - & & - & - & & - & & - & & - \\
\hline Bron et al ${ }^{15}$ & - & & - & - & & - & - & & - & & - & & - \\
\hline Buitelaar et $\mathrm{al}^{32}$ & 2 & & - & - & & - & - & & $5 \%$ & & - & & $4 \%$ \\
\hline Buitelaar et $\mathrm{al}^{65}$ & $4-5 * 2)$ & & - & - & & - & - & & $2 \%$ & & - & & $5 \%$ \\
\hline Casas et $\mathrm{a}^{33}$ & 5 & & - & - & & $8 \%$ & - & & $9 \%$ & & - & & $6 \%$ \\
\hline Fredriksen et $\mathrm{al}^{53}$ & - & & - & - & & Yes & Yes & & Yes & & - & & - \\
\hline Huss et al ${ }^{54,55}$ & 5 & & - & - & & - & - & & $5 \%$ & & - & & - \\
\hline Kooij et a $a^{45}$ & - & & - & - & & - & - & & - & & - & & - \\
\hline Valdizán-Usón et al ${ }^{62}$ & - & & - & - & & - & - & & - & & - & & - \\
\hline Amiri et $\mathrm{al}^{23}$ & - & & - & - & & - & - & & $13 \%$ & & - & & - \\
\hline Arabgol et $\mathrm{al}^{22}$ & - & & - & - & & - & - & & - & & - & & - \\
\hline Barbaresi et al ${ }^{18}$ & - & & - & - & & - & - & & - & & - & & - \\
\hline Barkley et al ${ }^{46}$ & - & & - & - & & - & - & & $58 \%$ & & - & & - \\
\hline Biederman et $\mathrm{a}^{21}$ & - & & - & - & & - & - & & Yes & & - & & - \\
\hline Cherland and & - & & - & - & & - & - & & - & & - & & - \\
\hline \multicolumn{14}{|l|}{ Fitzpatrick ${ }^{63}$} \\
\hline Döpfner et $\mathrm{al}^{56}$ & - & & - & - & & - & $3 \%$ & & - & & $1 \%$ & & $1 \%$ \\
\hline Efron et $\mathrm{al}^{47}$ & - & & - & - & & - & - & & $61 \%$ & & - & & - \\
\hline Findling et $\mathrm{al}^{24}$ & 3 & & $3 \%$ & - & & - & $3 \%$ & & - & & - & & - \\
\hline Garg et $\mathrm{al}^{57}$ & - & & - & - & & - & - & & - & & - & & - \\
\hline Gau et $\mathrm{al}^{27}$ & - & & - & - & & - & - & & $31 \%$ & & - & & - \\
\hline Ghuman et $\mathrm{al}^{16}$ & - & & - & - & & $11 \%$ & - & & $3 \%$ & & - & & - \\
\hline Greenhill et a ${ }^{26}$ & 2 & & - & - & & - & - & & - & & - & & - \\
\hline Haertling et al ${ }^{58}$ & - & & - & - & & $1 \%$ & $1 \%$ & & - & & $1 \%$ & & - \\
\hline Karabekiroglu et al ${ }^{59}$ & - & & - & - & & - & - & & - & & - & & - \\
\hline Kemner et $\mathrm{a}^{28}$ & 1 & & $1 \%$ & - & & - & $1 \%$ & & - & & - & & - \\
\hline Khajehpiri et a ${ }^{60}$ & - & & - & - & & $36 \%$ & - & & - & & - & & - \\
\hline Kratochvil et a $\left.\right|^{30}$ & 5 & & $5 \%$ & $12 \%$ & & - & - & & - & & $5 \%$ & & - \\
\hline Lee et $\mathrm{al}^{48}$ & - & & - & - & & - & - & & $38 \%$ & & - & & - \\
\hline Maayan et al ${ }^{17}$ & - & & - & - & & - & $9 \%$ & & - & & - & & - \\
\hline MTA group ${ }^{34-39}$ & - & & - & - & & - & - & & - & & - & & - \\
\hline Newcorn et a $\left.\right|^{25}$ & 5 & & - & - & & - & - & & - & & - & & - \\
\hline PATS group ${ }^{40-42}$ & $* 3)$ & & - & - & & - & $4 \%$ & & $3 \%$ & & - & & - \\
\hline Pelham et $\mathrm{al}^{49}$ & 2 & & - & $4 \%$ & & - & - & & - & & - & & - \\
\hline Smith et al ${ }^{19}$ & - & & - & - & & - & - & & - & & - & & - \\
\hline Ramtvedt et a ${ }^{64}$ & - & & - & - & & - & - & & $30 \%$ & & - & & - \\
\hline Robb et al ${ }^{|6|}$ & 5 & & - & - & & - & $6 \%$ & & - & & - & & - \\
\hline Silva et $\mathrm{al}^{14}$ & - & & - & - & & - & - & & - & & - & & - \\
\hline Steele et $\mathrm{a}^{66}$ & 10 & & - & - & & $11 \%$ & - & & - & & - & & - \\
\hline Stein et $\mathrm{al}^{50}$ & - & & - & - & & - & - & & $55 \%$ & & - & & - \\
\hline Swanson et $\mathrm{al}^{29}$ & 2 & & - & - & & - & - & & - & & - & & - \\
\hline Wigal et al ${ }^{44}$ & 5 & & - & - & & - & - & & - & & - & & - \\
\hline Zarinara et a ${ }^{143}$ & - & & - & - & & - & - & & - & & - & & - \\
\hline Total & - & & $1 \%-5 \%$ & $4 \%-12 \%$ & & $1 \%-36 \%$ & $1 \%-9 \%$ & & $2 \%-6$ & $1 \%$ & ।\%-5\% & & $1 \%-6 \%$ \\
\hline Placebo in total & - & & $0 \%-4 \%$ & $0 \%-3 \%$ & & $0 \%-1 \%$ & $0 \%-5 \%$ & & $1 \%-5$ & $8 \%$ & - & & $1 \%-5 \%$ \\
\hline Adult & - & & - & - & & $5 \%-8 \%$ & - & & $2 \%-$ & $3 \%$ & - & & $4 \%-6 \%$ \\
\hline Placebo adult & - & & - & - & & $1 \%$ & - & & $1 \%-7$ & & - & & $1 \%-5 \%$ \\
\hline Child & - & & $1 \%-5 \%$ & $4 \%-12 \%$ & & $1 \%-36 \%$ & $1 \%-9 \%$ & & $3 \%-6$ & $1 \%$ & $1 \%-5 \%$ & & $1 \%$ \\
\hline Placebo child & - & & $0 \%-4 \%$ & $0 \%-3 \%$ & & - & $0 \%-5 \%$ & & $1 \%-5$ & & - & & - \\
\hline Study & $\begin{array}{l}\text { Disinterested } \\
\text { in others }\end{array}$ & Diz & ziness & rowsiness & $\begin{array}{l}\text { Em } \\
\text { labi }\end{array}$ & $\begin{array}{l}\text { tional } \\
\text { ity }\end{array}$ & Euphoria & & $\begin{array}{l}\text { eling } \\
\text { ery }\end{array}$ & Irrit & ability & $\begin{array}{l}\text { Mood } \\
\text { alteration }\end{array}$ & $\begin{array}{ll} & \text { Nail } \\
\text { n } & \text { biting } \\
\end{array}$ \\
\hline Adler et $\mathrm{a}^{51}$ & - & - & - & & - & & - & 13 & & $8 \%$ & & - & - \\
\hline Adler et $\mathrm{a}^{31}$ & - & - & - & & - & & - & - & & $10 \%$ & & - & - \\
\hline Bejerot et a ${ }^{52}$ & - & $17 \%$ & - & & - & & - & - & & - & & $10 \%$ & - \\
\hline
\end{tabular}


Table 2 (Continued)

\begin{tabular}{|c|c|c|c|c|c|c|c|c|c|}
\hline Study & $\begin{array}{l}\text { Disinterested } \\
\text { in others }\end{array}$ & Dizziness & Drowsiness & $\begin{array}{l}\text { Emotional } \\
\text { lability }\end{array}$ & Euphoria & $\begin{array}{l}\text { Feeling } \\
\text { jittery }\end{array}$ & Irritability & $\begin{array}{l}\text { Mood } \\
\text { alteration }\end{array}$ & $\begin{array}{l}\text { Nail } \\
\text { biting }\end{array}$ \\
\hline Bron et al ${ }^{15}$ & - & - & - & - & - & - & - & - & - \\
\hline Buitelaar et $\mathrm{a}^{32}$ & - & $8 \%$ & - & - & - & - & $5 \%$ & - & - \\
\hline Buitelaar et $\mathrm{al}^{65}$ & - & - & - & - & - & - & $4 \%$ & - & - \\
\hline Casas et $\mathrm{al}^{33}$ & - & $12 \%$ & - & - & - & - & $5 \%$ & - & - \\
\hline Fredriksen et $\mathrm{a}^{53}$ & - & - & - & Yes & - & - & - & - & - \\
\hline Huss et a $\left.\right|^{54,55}$ & - & $6 \%$ & - & - & - & - & $6 \%$ & - & - \\
\hline Kooij et a $\left.\right|^{45}$ & - & $16 \%$ & & - & - & - & $15 \%$ & - & - \\
\hline Valdizán-Usón et al ${ }^{62}$ & - & - & - & - & - & - & - & - & - \\
\hline Amiri et $\mathrm{a}^{23}$ & - & - & - & - & - & - & $20 \%$ & - & - \\
\hline Arabgol et $\mathrm{al}^{22}$ & - & - & 0 & - & - & - & $6 \%$ & - & - \\
\hline Barbaresi et al ${ }^{18}$ & - & - & - & - & - & - & - & - & - \\
\hline Barkley et $\mathrm{al}^{46}$ & $43 \%$ & $10 \%$ & $25 \%$ & - & $43 \%$ & - & $66 \%$ & - & $29 \%$ \\
\hline Biederman et $\mathrm{a}^{21}$ & - & - & Yes & Yes & - & - & - & - & - \\
\hline Cherland and & - & - & - & - & - & - & - & - & - \\
\hline \multicolumn{10}{|l|}{ Fitzpatrick ${ }^{63}$} \\
\hline Döpfner et al ${ }^{56}$ & - & - & - & - & - & - & - & - & - \\
\hline Efron et a $\left.\right|^{47}$ & $31 \%$ & $12 \%$ & $18 \%$ & - & $28 \%$ & - & $80 \%$ & - & $45 \%$ \\
\hline Findling et $\mathrm{al}^{24}$ & - & - & - & $3 \%$ & - & - & $3 \%$ & - & - \\
\hline Garg et $\mathrm{al}^{57}$ & - & - & $3 \%$ & - & - & - & $6 \%$ & - & - \\
\hline Gau et $\mathrm{al}^{27}$ & $40 \%$ & - & $25 \%$ & - & $28 \%$ & - & $21 \%$ & - & $25 \%$ \\
\hline Ghuman et $\mathrm{al}^{16}$ & - & - & $18 \%$ & $29 \%$ & - & - & $29 \%$ & - & - \\
\hline Greenhill et $\mathrm{a}^{26}$ & - & - & - & $3 \%$ & - & - & $3 \%$ & - & - \\
\hline Haertling et $\mathrm{a}^{58}$ & - & $1 \%$ & - & $1 \%$ & - & - & - & $1 \%$ & - \\
\hline Karabekiroglu et al ${ }^{59}$ & - & - & - & - & - & - & - & - & - \\
\hline Kemner et $\mathrm{al}^{28}$ & - & $1 \%$ & - & $1 \%$ & - & - & $1 \%$ & $1 \%$ & - \\
\hline Khajehpiri et a ${ }^{60}$ & - & - & - & - & - & - & $57 \%$ & - & - \\
\hline Kratochvil et a ${ }^{30}$ & - & - & 0 & $5 \%$ & - & - & - & - & - \\
\hline Lee et $\mathrm{al}^{48}$ & $17 \%$ & $6 \%$ & $16 \%$ & - & $25 \%$ & - & $64 \%$ & - & $30 \%$ \\
\hline Maayan et al ${ }^{17}$ & - & - & $9 \%$ & $18 \%$ & - & - & - & - & - \\
\hline MTA group ${ }^{34-39}$ & - & - & - & - & - & - & - & - & - \\
\hline Newcorn et a $\left.\right|^{25}$ & - & - & - & - & - & - & $6 \%$ & - & - \\
\hline PATS group ${ }^{40-42}$ & - & - & Yes & $15 \%$ & - & - & $8 \%$ & $7 \%$ & - \\
\hline Pelham et $\mathrm{a}^{49}$ & - & $3 \%$ & - & - & - & - & - & - & - \\
\hline Smith et al ${ }^{19}$ & - & - & - & - & - & - & - & - & - \\
\hline Ramtvedt et al ${ }^{64}$ & $21 \%$ & $18 \%$ & $32 \%$ & - & $18 \%$ & - & $65 \%$ & - & - \\
\hline Robb et $\left.a\right|^{\mid 61}$ & - & $6 \%$ & - & $26 \%$ & - & - & $13 \%$ & - & - \\
\hline Silva et al ${ }^{14}$ & - & - & - & - & - & - & 0 & - & - \\
\hline Steele et $\mathrm{a}^{66}$ & - & - & - & $13 \%$ & - & - & - & - & - \\
\hline Stein et $\mathrm{al}^{50}$ & $34 \%$ & $11 \%$ & $28 \%$ & - & $16 \%$ & - & $71 \%$ & - & $22 \%$ \\
\hline Swanson et $\mathrm{al}^{29}$ & - & - & - & - & - & - & $1 \%$ & - & - \\
\hline Wigal et al ${ }^{44}$ & - & $9 \%$ & - & - & - & - & - & - & - \\
\hline Zarinara et $\mathrm{al}^{43}$ & - & - & - & - & - & - & - & - & - \\
\hline Total & $17 \%-41 \%$ & $1 \%-18 \%$ & $0 \%-32 \%$ & $1 \%-29 \%$ & $16 \%-43 \%$ & $13 \%$ & $0 \%-80 \%$ & $1 \%-10 \%$ & $22 \%-45 \%$ \\
\hline Placebo in total & $17 \%-36 \%$ & $0 \%-15 \%$ & $8 \%-53 \%$ & $0 \%-11 \%$ & $25 \%-45 \%$ & $2 \%$ & ।\%-80\% & $6 \%$ & $15 \%-29 \%$ \\
\hline Adult & - & $6 \%-17 \%$ & $0 \%-32 \%$ & - & - & $13 \%$ & $4 \%-15 \%$ & $10 \%$ & - \\
\hline Placebo, adult & - & $2 \%-7 \%$ & $8 \%-53 \%$ & - & - & $2 \%$ & $1 \%-23 \%$ & - & - \\
\hline Child & $17 \%-41 \%$ & $1 \%-18 \%$ & $0 \%-32 \%$ & $1 \%-29 \%$ & $16 \%-43 \%$ & - & $0 \%-80 \%$ & $1 \%-7 \%$ & $22 \%-45 \%$ \\
\hline Placebo, child & $17 \%-36 \%$ & $0 \%-15 \%$ & $8 \%-53 \%$ & $0 \%-11 \%$ & $25 \%-45 \%$ & - & $1 \%-80 \%$ & $6 \%$ & $15 \%-29 \%$ \\
\hline Study & Nervousness & Nightmares & $\begin{array}{l}\text { Prone to } \\
\text { crying }\end{array}$ & $\begin{array}{l}\text { Psychomotor } \\
\text { hyperactivity }\end{array}$ & Restlessness & Sadness & Staring & Talks less & Tics \\
\hline Adler et $\mathrm{al}^{51}$ & - & - & - & $5 \%$ & - & - & - & - & - \\
\hline Adler et $\mathrm{al}^{31}$ & - & - & - & - & - & - & - & - & - \\
\hline Bejerot et $\mathrm{a}^{52}$ & $12 \%$ & - & - & - & - & - & - & - & $1 \%$ \\
\hline Bron et al ${ }^{15}$ & $23 \%$ & - & - & - & - & - & - & - & - \\
\hline Buitelaar et $\mathrm{al}^{32}$ & $3 \%$ & - & - & - & $5 \%$ & - & - & - & - \\
\hline Buitelaar et $\mathrm{al}^{65}$ & - & - & - & - & $7 \%$ & - & - & - & - \\
\hline
\end{tabular}


Table 2 (Continued)

\begin{tabular}{|c|c|c|c|c|c|c|c|c|c|}
\hline Study & Nervousness & Nightmares & $\begin{array}{l}\text { Prone to } \\
\text { crying }\end{array}$ & $\begin{array}{l}\text { Psychomotor } \\
\text { hyperactivity }\end{array}$ & Restlessness & Sadness & Staring & Talks less & Tics \\
\hline Casas et $\mathrm{al}^{33}$ & - & - & - & - & $9 \%$ & - & - & - & - \\
\hline Fredriksen et $\mathrm{al}^{53}$ & - & - & - & - & - & - & - & - & Yes \\
\hline Huss et $\mathrm{al}^{54,55}$ & - & - & - & - & $4 \%$ & - & - & - & - \\
\hline Kooij et a $\left.\right|^{45}$ & - & - & - & - & - & - & - & - & $7 \%$ \\
\hline Valdizán-Usón et al ${ }^{62}$ & - & - & - & - & - & - & - & - & - \\
\hline Amiri et $\mathrm{a}^{23}$ & - & - & - & - & - & $20 \%$ & - & - & - \\
\hline Arabgol et $\mathrm{al}^{22}$ & - & - & - & - & - & - & - & - & - \\
\hline Barbaresi et al ${ }^{18}$ & - & - & - & - & - & - & - & - & - \\
\hline Barkley et $\mathrm{al}^{46}$ & - & $21 \%$ & $59 \%$ & $28 \%$ & - & $48 \%$ & $57 \%$ & $42 \%$ & - \\
\hline Biederman et $\mathrm{al}^{21}$ & - & - & - & - & - & - & - & - & - \\
\hline Cherland and & - & - & - & - & - & - & - & - & - \\
\hline \multicolumn{10}{|l|}{ Fitzpatrick ${ }^{63}$} \\
\hline Döpfner et al ${ }^{56}$ & - & - & - & - & - & $17 \%$ & - & - & $12 \%$ \\
\hline Efron et $\mathrm{a}^{47}$ & - & $21 \%$ & $71 \%$ & - & - & $56 \%$ & $62 \%$ & $28 \%$ & $28 \%$ \\
\hline Findling et $\mathrm{al}^{24}$ & - & - & - & - & - & - & - & - & 0 \\
\hline Garg et $\mathrm{a}^{57}$ & - & - & - & - & - & - & - & - & - \\
\hline Gau et $\mathrm{al}^{27}$ & - & $25 \%$ & $47 \%$ & - & - & - & $31 \%$ & $31 \%$ & $18 \%$ \\
\hline Ghuman et $\mathrm{al}^{16}$ & - & - & $29 \%$ & $3 \%$ & - & - & - & - & - \\
\hline Greenhill et $\mathrm{a}^{26}$ & - & - & - & - & - & - & - & - & - \\
\hline Haertling et $\mathrm{a}^{58}$ & - & - & - & - & - & - & - & - & - \\
\hline Karabekiroglu et $\mathrm{a}^{59}$ & - & - & - & - & - & - & - & - & - \\
\hline Kemner et $\mathrm{al}^{28}$ & - & - & $1 \%$ & - & - & - & - & - & - \\
\hline Khajehpiri et a $\left.\right|^{60}$ & - & $24 \%$ & - & - & - & - & - & - & $35 \%$ \\
\hline Kratochvil et a $\left.\right|^{30}$ & $10 \%$ & - & - & - & - & - & - & - & - \\
\hline Lee et $\mathrm{al}^{48}$ & - & $13 \%$ & $49 \%$ & - & - & $38 \%$ & $35 \%$ & $27 \%$ & $17 \%$ \\
\hline Maayan et $\mathrm{al}^{17}$ & - & - & - & - & - & - & - & - & - \\
\hline MTA group ${ }^{34-39}$ & - & - & - & - & - & - & - & - & - \\
\hline Newcorn et $\mathrm{al}^{25}$ & - & - & - & - & - & - & - & - & - \\
\hline PATS group ${ }^{40-42}$ & - & - & - & - & - & - & - & - & - \\
\hline Pelham et al ${ }^{49}$ & - & - & - & $5 \%$ & - & - & - & - & - \\
\hline Smith et a $\left.\right|^{19}$ & - & - & - & - & - & - & - & - & - \\
\hline Ramtvedt et $\mathrm{a}^{64}$ & - & $9 \%$ & $65 \%$ & - & - & $41 \%$ & $21 \%$ & $29 \%$ & $15 \%$ \\
\hline Robb et $\mathrm{a}^{61}$ & - & - & - & - & - & - & - & - & - \\
\hline Silva et al ${ }^{14}$ & - & - & - & - & - & - & - & - & - \\
\hline Steele et $\mathrm{al}^{66}$ & - & $13 \%$ & - & - & - & - & - & - & - \\
\hline Stein et $\mathrm{a}^{50}$ & - & $17 \%$ & $54 \%$ & - & - & $47 \%$ & $54 \%$ & $32 \%$ & $17 \%$ \\
\hline Swanson et $\mathrm{a}^{29}$ & - & - & - & - & - & - & - & - & - \\
\hline Wigal et al ${ }^{44}$ & - & - & - & - & - & - & - & - & - \\
\hline Zarinara et $\mathrm{a}^{43}$ & - & - & - & - & $26 \%$ & - & - & - & - \\
\hline Total & $3 \%-23 \%$ & $9 \%-25 \%$ & $1 \%-7 \mid \%$ & $3 \%-28 \%$ & $4 \%-26 \%$ & $0 \%-56 \%$ & $21 \%-62 \%$ & $27 \%-42 \%$ & $0 \%-35 \%$ \\
\hline Placebo in total & $1 \%$ & $12 \%-20 \%$ & $38 \%-49 \%$ & $0 \%-7 \%$ & $0 \%-4 \%$ & $31 \%-50 \%$ & $30 \%-68 \%$ & $15 \%-38 \%$ & $2 \%-30 \%$ \\
\hline Adult & $3 \%-23 \%$ & - & - & $5 \%$ & $4 \%-9 \%$ & - & - & - & $1 \%$ \\
\hline Placebo, adult & $1 \%$ & - & - & $7 \%$ & $0 \%-4 \%$ & - & - & - & $2 \%$ \\
\hline Child & $10 \%$ & $9 \%-25 \%$ & $1 \%-71 \%$ & $3 \%-28 \%$ & $26 \%$ & $0 \%-47 \%$ & $21 \%-62 \%$ & $27 \%-42 \%$ & $0 \%-35 \%$ \\
\hline Placebo, child & - & $12 \%-20 \%$ & $38 \%-49 \%$ & - & - & $31 \%-50 \%$ & $30 \%-68 \%$ & $15 \%-38 \%$ & $4 \%-30 \%$ \\
\hline
\end{tabular}

Notes: *1) "most frequently recorded". *2) 5 (recorded in open-label), 4 (recorded in withdrawal); *3) 15 most frequently recorded adverse events.

Three studies mentioned when the symptoms started. Adler et $\mathrm{al}^{51}$ reported that most of the symptoms occurred within the first month of therapy. In Adler et al, ${ }^{31} 82.4 \%$ of symptoms were reported during the first 30 days. Arabgol et al reported that the symptoms occurred 1-2 weeks after the medication was started. ${ }^{22}$ None of the studies provided information on when the side effects started exactly, except Robb et al, who reported two severe side effects: one at 18 days, and one at 9 days. ${ }^{61}$ Biederman et al stated that the same side effects were reported in treatment and in placebo washout. ${ }^{21}$ Three studies failed to offer any information about the time the symptoms occurred..$^{19,24,34-39}$

\section{Are the reported side effects reversible?}

Most of the studies failed to provide information on whether side effects persisted following discontinuation of the drug. 
In seven of the studies, patients were followed up after the end of the medication. ${ }^{18,19,25,33-42}$ However, none of these allowed conclusions to be drawn regarding the reversibility of reported side. Cherland and Fitzpatrick were vague in this respect, stating simply that "most" participants improved following withdrawal of the medication. ${ }^{63}$ Adler et al describes the symptoms experienced by some of the participants as reversible. ${ }^{31}$ In three of the studies, all reported side effects disappeared following withdrawal of the medication. ${ }^{17,46,47}$ Two of the studies report that the side effects persisted until the end of the study. ${ }^{56,58}$

\section{Discussion}

\section{Study design and selection of studies}

Does methylphenidate cause changes in behavior? Current prescribing practices mean that many patients start taking methylphenidate as children and continue treatment until they reach adulthood. Five of the studies selected specifically recorded and discussed changes in behavior. ${ }^{31,40-42,59,63,64}$ Unfortunately, these studies cannot be compared in terms of quality (Table 3).

\section{Rates of withdrawal due to side effects}

In 29 of the studies, participants withdrew early because of side effects. Withdrawal rates ranged from 0 to $27 \%$, and six of the studies failed to provide this information at all. Three of the studies ${ }^{27,29,49}$ - two with a partial focus on the safety of methylphenidate 27,29 - stipulated that participants had to be receiving a stable dose of methylphenidate prior to entry into the study. All three reported zero withdrawal rates. Inclusion criteria for 13 of the studies stipulated that participants had to be methylphenidate-naïve. Three of these ${ }^{23,45,60}$ reported zero withdrawal rates, while one ${ }^{19}$ failed to report this information. Maayan et al recorded the highest withdrawal rate $-27 \% .{ }^{17}$ However, the study's eleven participants did not constitute a representative sample. For studies that included only methylphenidate-naïve participants, the mean withdrawal rate was $6.7 \%$. Studies that included only participants able to tolerate methylphenidate or that did not establish whether participants had prior exposure to the drug recorded a mean withdrawal rate of $7.4 \%$. The reviewed data would thus suggest that there is no link between prior exposure to methylphenidate and early withdrawal due to side effects.

\section{Changes in behavior}

The occurrence of recorded changes in behavior differ widely. The symptom "irritability" was recorded by no subjects $^{14}$ to $80 \%$ of subjects. ${ }^{47}$ In every case of the most frequently recorded symptoms, Efron et al recorded the highest percentages: "prone to crying" (71\%), "staring" (62\%), "anxiety" (61\%), "sadness" (56\%), "nail biting" (45\%), "euphoria" (43\%), and "talks less" (42\%). ${ }^{47}$

\section{Occurrence of side effects}

While Khajehpiri et al reported side effects for all their participants, ${ }^{60}$ Haertling et al reported the lowest rate of occurrence of side effects $(13.7 \%) .{ }^{58}$ Studies with methylphenidate-naïve participants reported side effects in $67.7 \%$ of participants. Studies that did not require participants to be methylphenidate-naïve or required them to be able to tolerate methylphenidate recorded a side effect rate of $57.7 \%$. What was the reason for the low rate of occurrence reported in Haertling et al $?^{58}$ The study was similar to the other studies

Table 3 Studies that recorded and discussed changes in behavior

\begin{tabular}{|c|c|c|c|c|c|}
\hline Study & Adler et $\mathrm{al}^{3 !}$ & $\begin{array}{l}\text { Cherland and } \\
\text { Fitzpatrick }^{63}\end{array}$ & $\begin{array}{l}\text { Karabekiroglu } \\
\text { et al }{ }^{59}\end{array}$ & PATS group ${ }^{40-42}$ & Ramtvedt et al $^{64}$ \\
\hline Study design & Open-label & Retrospective & Open-label & Crossover, parallel & Crossover \\
\hline Treatment period & $6-12$ months & 21 months & 15 days & 70 weeks & 6 weeks \\
\hline Follow-up & No & No & No & Yes & No \\
\hline Patients completed & 550 & 98 & 90 & 165 & 34 \\
\hline Age, years & $18-65$ & $4-17$ & $5-15$ & $3.5-5$ & II.3 \\
\hline Placebo group & No & No & No & Yes & Yes \\
\hline Methylphenidate-naïve & No & Not known & Yes & Yes & Yes \\
\hline Limit for symptoms & $10 \%$ & Not known & Not known & 15 most frequent & Not known \\
\hline Changes in behavior & $1.8 \%$ & Single cases & No & Yes & Yes \\
\hline Focus of study & $\begin{array}{l}\text { Changes in behavior in } \\
\text { relation to long-term } \\
\text { safety of methylphenidate }\end{array}$ & $\begin{array}{l}\text { Psychotic } \\
\text { side effects of } \\
\text { methylphenidate }\end{array}$ & $\begin{array}{l}\text { Short-term } \\
\text { side effects of } \\
\text { methylphenidate }\end{array}$ & $\begin{array}{l}\text { Safety and } \\
\text { tolerability of } \\
\text { methylphenidate }\end{array}$ & $\begin{array}{l}\text { Use of methylphenidate } \\
\text { in minimizing adverse } \\
\text { events }\end{array}$ \\
\hline
\end{tabular}

Notes: For Adler et al, changes in behavior were mentioned in the article, but are not shown in the tables. ${ }^{31}$ For Cherland and Fitzpatrick, it was impossible to determine with certainty whether the behavioral changes observed were reversible. ${ }^{63}$ The Karabekiroglu et al study was specifically designed to study short-term effects. ${ }^{59}$ Subjects in the PATS group studies used methylphenidate off-label because of their age. ${ }^{40-42}$ In Ramtvedt et al, methylphenidate treatment was limited to 2 weeks. ${ }^{64}$ 
in terms of age distribution and number of participants. Nineteen percent of participants received methylphenidate for the first time. The remaining participants were already using methylphenidate, but in a different form. The study was funded by the pharmaceutical industry. If both the comparability of studies and the evaluation of side effects are to be improved, future studies will need to exclude participants with prior exposure to methylphenidate.

Barkley et al posited that any behavioral changes observed during their study had to be linked to the condition under study. ${ }^{46}$ They supported this assertion by stating that the same symptoms occurred during initial testing and during the placebo phase of the trial, and that the physiological effects of methylphenidate disappeared within 24 hours of administration. The authors' assertion should be treated with caution. Participants were aged between 5 and 13 years. Inclusion criteria stipulated that participants had to have developed ADHD symptoms before the age of 7 years. While no such information was provided in the article, it must be assumed that some of the participants would have been receiving drug-based treatment for a number of years prior to enrollment. The assertion that side effects cease completely 24 hours after administration directly contradicts results from animal studies, which suggest that exposure leads to structural changes inside the brain, as well as clear changes in behaviors displayed by adult rats. ${ }^{2,3}$

\section{Study funding and its impact on results}

Some of the articles mentioned relevant results, but failed to discuss these further. Adler et al mentioned specifically instructing participants to report psychotic side effects, and that participants reported mania, aggression, and paranoia. ${ }^{51}$ However, during their presentation of results, the authors introduced the rule that only "symptoms occurring in at least $10 \%$ of participants" were to be classed as side effects. As psychiatric side effects only occurred in $1.8 \%$ of participants, these were not discussed any further. The study was conducted in conjunction with the pharmaceutical industry. The study's first author declared a conflict of interest. Casas et al reported that one participant attempted to commit suicide, but failed to provide any further details. ${ }^{33}$ It is impossible to establish whether side effects occurred during the washout period. The authors merely observed that no more than three participants from each arm of the study reported any side effects in the week between outcome measurements and follow-up visit. This rather vague statement leaves room for speculation. The study was funded by the pharmaceutical industry.

\section{Are the reported side effects reversible?}

Are reported side effects reversible once administration is discontinued? Only a few of the details provided in the studies can be verified. Even studies aimed at testing the safety of methylphenidate did not provide information on the cessation of side effects. Both Döpfner et al and Haertling et al reported side effects as not reversible until the end of the study. ${ }^{56,58}$ Only Barkley et al and Efron et al provided clear information in support of the statement that side effects were completely reversible. ${ }^{46,47}$ The other studies failed to provide information on this issue.

\section{Lack of data}

With the exception of the studies mentioned earlier, none of the studies discussed the issue of behavioral change. While many of the articles listed instances of behavioral change, the tables provided only listed side effects reported by a certain percentage of respondents (up to 10\%). This of course raises the question of how many examples of behavioral change remain undetected among studies that only list side effects above a certain minimum frequency. Whether behavioral side effects are particularly common is of no real relevance. The fact that there is a real risk of such side effects developing should be reason enough to instill a sense of caution when prescribing methylphenidate. What is more, data from the reviewed studies do not rule out the possibility that the observed side effects may be irreversible. The MTA study may be able to provide some indirect answers. ${ }^{34-39}$ When compared with children from the control group, children enrolled in the MTA study were ten times more likely to be admitted to psychiatric institutions.

\section{Future studies}

With the exception of Cherland and Fitzpatrick, which was retrospective in design, none of the studies set out specifically and exclusively to study changes in behavior. ${ }^{63}$ What is more, most of the studies were too short to allow inferences to be made regarding the drug's long-term effects. As the majority of the studies did not include follow-up examinations, it is impossible to determine whether behavioral symptoms were reversible.

It would therefore be desirable if future studies were to meet the required quality criteria as determined by study design (multicenter, randomized, double-blind, placebocontrolled), and if they were designed to ensure a study duration long enough to permit the study of long-term effects. It would also be desirable if future studies were to include a follow-up phase, as this would allow conclusions to be drawn 
regarding the reversibility or irreversibility of behavioral effects. In terms of inclusion criteria, it would be desirable if studies were to be limited to participants not previously exposed to methylphenidate. This would allow potential side effects to be evaluated with regard to both frequency and severity. Future studies should set out to study changes in behavior or at the very least side effects in general. The use of assessment tools would improve comparability between studies by allowing side effects to be both recorded and evaluated. Funding by pharmaceutical companies should always be treated with caution.

The question of whether methylphenidate treatment is associated with behavioral effects remains to be fully elucidated. What is clear, however, is that children and adolescents continue to be treated with substances regulated by the Narcotics Drug Act, and that they are often exposed to these drugs for many years, and at a time when their brains are still growing and therefore vulnerable. The fact that the long-term safety of these drugs remains to be fully elucidated means that there is an urgent need for further research. In April 2016, Inglis et al published the study protocol of the ADDUCE study, a prospective observational study to investigate long-term adverse effects of methylphenidate in children and adolescents with ADHD, funded by the European Commission. Hopefully, this study can offer some security for the further use of methylphenidate. ${ }^{67}$

\section{Summary and conclusion}

In Germany, the use of methylphenidate is regulated by the Narcotic Drugs Act (Betäubungsmittelgesetz). Among other disorders, methylphenidate is used in the treatment of children and adolescents with ADHD. Since 2011, the drug has been licensed for use in adults with ADHD.

The fact that methylphenidate is often used over many years raises the following questions. Does methylphenidate lead to changes in behavior either during or after treatment? At what point do changes in behavior occur? Has there been sufficient research into the side effects of treatment with methylphenidate, and does this research include the period after treatment is discontinued?

An analysis of the articles included in the list of references provides the following answers. Seven studies specifically set out to study, record, and discuss changes in behavior. ${ }^{31,40-42}$ ${ }^{59,63,64}$ Eight studies did not set out to study behavioral effects, but recorded and discussed them. ${ }^{16,32,45,46,48,50,52,61}$ A total of 28 studies recorded behavioral effects, but failed to discuss these further. ${ }^{14,15,17,19,21-30,32-39,44,47,49,51,53-58,60,66}$ Three studies did not include behavioral effects. . $^{18,43,62}$
Findings from our review of the selected studies can be summarized as follows. While there is some evidence to suggest that methylphenidate causes changes in behavior, the majority of the studies reviewed paid little or no attention to this issue. Based on the available data, it is impossible to determine the point at which such behavioral effects occur. The frequency of occurrence of behavioral effects is also impossible to determine with certainty. Based on the available data, it is not possible to rule out whether behavioral effects may persist or not persist once treatment is discontinued.

There is a relative dearth of studies dealing with the long-term outcomes of methylphenidate treatment and the issue of posttreatment follow-up. As a result, this literature review was unable to establish a good safety profile for the drug. Further studies are needed, which in order to provide meaningful data will need to meet certain quality criteria. Aside from being randomized, placebo-controlled, and double-blind multicenter studies, they will need to include a sufficient number of participants. They will also need to be of sufficient duration to allow conclusions regarding the long-term effects of methylphenidate. Participants should be methylphenidate-naïve. A follow-up phase must be included if the reversibility of behavioral effects is to be studied. In conclusion, despite countless publications and extensive administration, especially to children, we have insufficient data to judge the long-term effects and risks of taking methylphenidate.

\section{Acknowledgment}

This paper contains results from a thesis by Doris S KonradBindl - "Führt Methylphenidat zu Wesensänderungen? Eine Literaturstudie" - submitted to the Faculty of Medicine of Ludwig-Maximilians-Universität München in fulfillment of the requirement for a medicine degree.

\section{Disclosure}

The authors report no conflicts of interest in this work.

\section{References}

1. Britton GB. Cognitive and emotional behavioral changes associated with methylphenidate treatment: a review of preclinical studies. Int $J$ Neuropsychopharmacol. 2012;15(1):41-53.

2. Carlezon WA, Mague SD, Andersen SL. Enduring behavioral effects of early exposure to methylphenidate in rats. Biol Psychiatry. 2003;54(12): 1330-1337.

3. Bolaños CA, Barrot M, Berton O, Wallace-Black D, Nestler EJ. Methylphenidate treatment during pre- and periadolescence alters behavioral responses to emotional stimuli at adulthood. Biol Psychiatry. 2003;54(12):1317-1329.

4. Volkow ND, Wang GJ, Fowler JS, et al. Dopamine transporter occupancies in the human brain induced by therapeutic doses of oral methylphenidate. Am J Psychiatry. 1998;155(10):1325-1331. 
5. Wang GJ, Volkow ND, Wigal T, et al. Long-term stimulant treatment affects brain dopamine transporter level in patients with attention deficit hyperactive disorder. PLoS One. 2013;8(5):1-6.

6. Advokat $\mathrm{C}$. What are the cognitive effects of stimulant medications? Emphasis on adults with attention-deficit/hyperactivity disorder (ADHD). Neurosci Biobehav Rev. 2010;34(8):1256-1266.

7. Storebø OJ, Ramstad E, Krogh HB, et al. Methylphenidate for children and adolescents with attention deficit hyperactivity disorder (ADHD). Cochrane Database Syst Rev. 2015;(11):CD009885.

8. Reiche D. Roche Lexikon Medizin [Medical Encyclopedia]. 4th ed. Munich: Urban \& Fischer; 2003.

9. Challman T, Lipsky JJ. Methylphenidate: its pharmacology and uses. Mayo Clin Proc. 2000;75(7):711-721.

10. Kuczenski R, Segal D. Effects of methylphenidate on extracellular dopamine, serotonin, and norepinephrine: comparison with amphetamine. J Neurochem. 1997;68(5):2032-2037.

11. Gatley SJ, Pan D, Chen R, Chaturvedi G, Ding YS. Affinities of methylphenidate derivatives for dopamine, norepinephrine and serotonin transporters. Life Sci. 1996;58(12):231-239.

12. Gray JD, Punsoni M, Tabori NE, et al. Methylphenidate administration to juvenile rats alters brain areas involved in cognition, motivated behaviors, appetite, and stress. J Neurosci. 2007;27(27):7196-7207.

13. Germany Federal Ministry of Health. Bekanntmachung eines Beschlusses des Gemeinsamen Bundesausschusses über eine Änderung der Arzneimittel-Richtlinie [Declaration of a resolution of the Federal Joint Committee about a modification of the guideline for medicine]. Berlin: Bundesministerium für Gesundheit; 2010.

14. Silva RR, Muniz R, Pestreich L, et al. Efficacy and duration of effect of extended-release dexmethylphenidate versus placebo in schoolchildren with attention-deficit/hyperactivity disorder. J Child Adolesc Psychopharmacol. 2006;16(3):239-251.

15. Bron TI, Bijlenga D, Boonstra AM, et al. OROS-methylphenidate efficacy on specific executive functioning deficits in adults with ADHD: a randomized, placebo-controlled cross-over study. Eur Neuropsychopharmacol. 2014;24(4):519-528.

16. Ghuman JK, Ginsburg GS, Subramaniam G, Human HS, Kau AS, Riddle MA. Psychostimulants in preschool children with attention-deficit/ hyperactivity disorder: clinical evidence from a developmental disorders institution. J Am Acad Child Adolesc Psychiatry. 2001;40(5):516-524.

17. Maayan L, Paykina N, Fried J, Strauss T, Gugga SS, Greenhill L. The open-label treatment of attention-deficit/hyperactivity disorder in 4- and 5-year-old children with beaded methylphenidate. $J$ Child Adolesc Psychopharmacol. 2009;19(2):147-153.

18. Barbaresi WJ, Katusic SK, Colligan RC, Weaver AL, Leibson CL, Jacobsen SJ. Long-term stimulant medication treatment of attentiondeficit/hyperactivity disorder: results from a population-based study. J Dev Behav Pediatr. 2006;27(1):1-10.

19. Smith G, Jongeling B, Hartmann P. Raine ADHD Study: Long-Term Outcomes Associated with Stimulant Medication in the Treatment of ADHD in Children. Perth: Government of Western Australia Department of Health; 2010.

20. Aagaard L, Hansen EH. The occurrence of adverse drug reactions reported for attention deficit hyperactivity disorder (ADHD) medications in the pediatric population: a qualitative review of empirical studies. Neuropsychiatr Dis Treat. 2011;7:729-744.

21. Biederman J, Quinn D, Weiss M, et al. Efficacy and safety of Ritalin LA, a new, once daily, extended-release dosage form of methylphenidate, in children with attention deficit hyperactivity disorder. Paediatr Drugs. 2003;5(12):833-841.

22. Arabgol F, Panaghi L, Hebrani P. Reboxetine versus methylphenidate in treatment of children and adolescents with attention deficit-hyperactivity disorder. Eur Child Adolesc Psychiatry. 2009;18(1):53-59.

23. Amiri S, Mohammadi MR, Mohammadi M, Neuroozinejad GH, Kahbazi M, Akhondzadeh S. Modafinil as a treatment for attentiondeficit/hyperactivity disorder in children and adolescents: a double blind, randomized clinical trial. Prog Neuropsychopharmacol Biol Psychiatry. 2008;32(1):145-149.
24. Findling RL, Quinn D, Hatch SJ, Cameron SJ, DeCory HH, McDowell M. Comparison of the clinical efficacy of twice-daily Ritalin and once-daily Equasym XL with placebo in children with attention deficit/hyperactivity disorder. Eur Child Adolesc Psychiatry. 2006; 15(8):450-459.

25. Newcorn JH, Kratochvil CJ, Allen AJ. Atomoxetine and osmotically released methylphenidate for the treatment of attention deficit hyperactivity disorder: acute comparison and differential response. $\mathrm{Am} \mathrm{J}$ Psychiatry. 2008;165(6):721-730.

26. Greenhill L, Muniz R, Ball RR, Levine A, Pestreich L, Jiang H. Efficacy and safety of dexmethylphenidate extended-release capsules in children with attention-deficit/hyperactivity disorder. $J$ Am Acad Child Adolesc Psychiatry. 2006;45(7):817-823.

27. Gau SS, Shen HY, Soong WT, Gau CS. An open-label, randomized, active-controlled equivalent trial of osmotic release oral system methylphenidate in children with attention-deficit/hyperactivity disorder in Taiwan. J Child Adolesc Psychopharmacol. 2006;16(4): 441-455.

28. Kemner JE, Starr HL, Ciccone PE, Hooper-Wood CG, Crockett RS. Outcomes of OROS methylphenidate compared with atomoxetine in children with ADHD: a multicenter, randomized prospective study. Adv Ther. 2005;22(5):498-512.

29. Swanson JM, Wigal SB, Wigal T, et al. A comparison of once-daily extended-release methylphenidate formulations in children with attention-deficit/hyperactivity disorder in the laboratory school (the COMACS study). Pediatrics. 2004;113(3 Pt 1):e206-e216.

30. Kratochvil CJ, Heiligenstein JH, Dittmann R, et al. Atomoxetine and methylphenidate treatment in children with ADHD: a prospective, randomized, open-label trial. J Am Acad Child Adolesc Psychiatry. 2002; 41(7):776-784.

31. Adler LA, Orman C, Starr HL, et al. Long-term safety of OROS methylphenidate in adults with attention-deficit/hyperactivity disorder: an open-label, dose-titration, 1-year study.J Clin Psychopharmacol. 2011; 31(1):108-114.

32. Buitelaar JK, Ramos-Quiroga A, Casas M, et al. Safety and tolerability of flexible dosages of prolonged-release OROS methylphenidate in adults with attention-deficit/hyperactivity disorder. Neuropsychiatr Dis Treat. 2009;5:457-466.

33. Casas M, Rösler M, Kooij JJ, et al. Efficacy and safety of prolongedrelease OROS methylphenidate in adults with attention deficit/ hyperactivity disorder: a 13-week, randomized, double-blind, placebocontrolled, fixed-dose study. World J Biol Psychiatry. 2013;14(4): 268-281.

34. The MTA Cooperative Group. A 14-month randomized clinical trial of treatment strategies for attention-deficit/hyperactivity disorder. Arch Gen Psychiatry. 1999;56(12):1073-1086.

35. Arnold LE, Howard BA, Cantwell DP, et al. National Institute of Mental Health collaborative multimodal treatment study of children with ADHD (the MTA). Arch Gen Psychiatry. 1997;54(9): 865-870.

36. MTA Cooperative Group. National Institute of Mental Health multimodal treatment study of ADHD follow-up: 24-month outcomes of treatment strategies for attention-deficit/hyperactivity disorder. Pediatrics. 2004;113(4):754-761

37. MTA Cooperative Group. National Institute of Mental Health multimodal treatment study of ADHD follow-up: changes in effectiveness and growth after the end of treatment. Pediatrics. 2004;113(4): 762-769.

38. Jensen PS, Arnold LE, Swanson JM, et al. 3-Year follow-up of the NIMH MTA study. J Am Acad Child Adolesc Psychiatry. 2007;6(8):989-1002.

39. Molina BS, Hinshaw SP, Swanson JM, et al. The MTA at 8 years: prospective follow-up of children treated for combined type ADHD in a multisite study. J Am Acad Child Adolesc Psychiatry. 2009;48(5): 484-500.

40. Greenhill L, Kollins S, Abikoff H, et al. Efficacy and safety of immediaterelease methylphenidate treatment for preschoolers with ADHD. $J$ Am Acad Child Adolesc Psychiatry. 2006;45(11):1284-1293. 
41. Wigal T, Greenhill L, Chuang S, et al. Safety and tolerability of methylphenidate in preschool children with ADHD. J Am Acad Child Adolesc Psychiatry. 2006;45(11):1294-1303.

42. Riddle MA, Yershova K, Lazzaretto D, et al. The Preschool AttentionDeficit/Hyperactivity Disorder Treatment Study (PATS) 6-year followup. J Am Acad Child Adolesc Psychiatry. 2013;52(3):264-278.

43. Zarinara AR, Mohammadi MR, Hazrati N, et al. Venlafaxine versus methylphenidate in pediatric outpatients with attention deficit hyperactivity disorder: a randomized, double-blind comparison trial. Hum Psychopharmacol. 2010;25(7-8):530-535.

44. Wigal SB, Nordbrock E, Adjei AL, Childress A, Kupper RJ, Greenhill L. Efficacy of methylphenidate hydrochloride extended-release capsules (Aptensio XR) in children and adolescents with attention-deficit/ hyperactivity disorder: a phase III, randomized, double-blind study. CNS Drugs. 2015;29(4):331-340.

45. Kooij JJ, Burger H, Boonstra AM, Van der Linden PD, Kalma LE, Buitelaar JK. Efficacy and safety of methylphenidate in 45 adults with attention-deficit/hyperactivity disorder: a randomized placebo-controlled double-blind cross-over trial. Psychol Med. 2004;34(6):973-982.

46. Barkley RA, McMurray MB, Edelbrock CS, Robbins K. Side effects of methylphenidate in children with attention deficit hyperactivity disorder: a systemic-placebo-controlled evaluation. Pediatrics. 1990;86(2): 184-192.

47. Efron D, Jarman F, Barker M. Side effects of methylphenidate and dexamphetamine in children with attention deficit hyperactivity disorder: a double-blind, crossover trial. Pediatrics. 1997;100(4):662-666.

48. Lee J, Grizenko N, Bhat V, Sengputa S, Polotskaia A, Joober R. Relation between therapeutic response and side effects induced by methylphenidate as observed by parents and teachers of children with ADHD. BMC Psychiatry. 2011;11:70.

49. Pelham WE, Gnagy EM, Burrows-Maclean L, et al. Once-a-day Concerta methylphenidate versus three-times-daily methylphenidate in laboratory and natural settings. Pediatrics. 2001;107(6):E105.

50. Stein MA, Sarampote CS, Waldman ID, et al. A dose-response study of OROS methylphenidate in children with attention-deficit/hyperactivity disorder. Pediatrics. 2003;112(5):e404-e413.

51. Adler LA, Spencer T, McGough JJ, Jiang H, Muniz R. Long-term effectiveness and safety of dexmethylphenidate extended-release capsules in adult ADHD. J Atten Disord. 2009;12(5):449-459.

52. Bejerot S, Rydén EM, Arlinde CM. Two-year outcome of treatment with central stimulant medication in adult attention-deficit/hyperactivity disorder: a prospective study. J Clin Psychiatry. 2010;71(12): 1590-1597.

53. Fredriksen M, Dahl AA, Martinsen EW, Klungsoyr O, Haavik J, Peleikis DE. Effectiveness of one-year pharmacological treatment of adult attention-deficit/hyperactivity disorder (ADHD): an open-label prospective study of time in treatment, dose, sideeffects and comorbidity. Eur Neuropsychopharmacol. 2014;24(12): 1873-1884.

54. Huss M, Ginsberg Y, Tvedten T, et al. Methylphenidate hydrochloride modified-release in adults with attention deficit hyperactivity disorder: a randomized double-blind placebo-controlled trial. Adv Ther. 2014; 31(1):44-65
55. Huss M, Ginsberg Y, Arngrim T, et al. Open-label dose optimization of methylphenidate modified release long acting (MPH-LA): a post hoc analysis of real-life titration from a 40-week randomized trial. Clin Drug Investig. 2014;34(9):639-649.

56. Döpfner M, Görtz-Dorten A, Breuer D, Rothenberger A. An observational study of once-daily modified release methylphenidate in ADHD: effectiveness on symptoms and impairment, and safety. Eur Child Adolesc Psychiatry. 2011;20(2):243-255.

57. Garg J, Arun P, Chavan BS. Comparative short term efficacy and tolerability of methylphenidate and atomoxetine in attention deficit hyperactivity disorder. Indian Pediatr. 2014;51(7):550-554.

58. Haertling F, Mueller B, Bilke-Hentsch O. Effectiveness and safety of a long-acting, once-daily, two-phase release formulation of methylphenidate (Ritalin LA) in school children under daily practice conditions. Atten Defic Hyperact Disord. 2015;7(2):157-164.

59. Karabekiroglu K, Yazgan YM, Dedeoglu C. Can we predict short-term side effects of methylphenidate immediate-release? Int J Psychiatry Clin Pract. 2008;12(1):48-54

60. Khajehpiri Z, Mahmoudi-Gharaei J, Faghihi T, Karimzadeh I, Khalili H, Mohammadi M. Adverse reactions of methylphenidate in children with attention deficit-hyperactivity disorder: report from a referral center. J Res Pharm Pract. 2015;3(4):130-136.

61. Robb AS, Findling RL, Childress AC, Berry SA, Belden HW, Wigal SB. Efficacy, safety, and tolerability of a novel methylphenidate extendedrelease oral suspension (MEROS) in ADHD. J Atten Disord. Epub 2014 May 29.

62. Valdizán-Usón JR, Cánovas-Martínez A, De Lucas-Taracena MT, et al. A response to methylphenidate by adult and pediatric patients with attention-deficit/hyperactivity disorder: the Spanish multicenter DIHANA study. Neuropsychiatr Dis Treat. 2013;9:211-218.

63. Cherland E, Fitzpatrick R. Psychotic side effects of psychostimulants: a 5-year review. Can J Psychiatry. 1999;44(8):811-813.

64. Ramtvedt BE, Aabech HS, Sundet K. Minimizing adverse events while maintaining clinical improvement in a pediatric attention-deficit/ hyperactivity disorder crossover trial with dextroamphetamine and methylphenidate. J Child Adolesc Psychopharmacol. 2014;24(3): 130-139.

65. Buitelaar JK, Trott GE, Hofecker M, et al. Long-term efficacy and safety outcomes with OROS-MPH in adults with ADHD. Int $J$ Neuropsychopharmacol. 2012;15(1):1-13.

66. Steele M, Weiss M, Swanson J, Wang J, Prinzo RS, Binder CE. A randomized, controlled, effectiveness trial of OROS-methylphenidate compared to usual care with immediate-release methylphenidate in attention deficit-hyperactivity disorder. Can J Clin Pharmacol. 2006;13(1):e50-e62.

67. Inglis SK, Carucci S, Garas P, et al. Prospective observational study protocol to investigate long-term adverse effects of methylphenidate in children and adolescents with ADHD: the Attention Deficit Hyperactivity Disorder Drugs Use Chronic Effects (ADDUCE) Study. BMJ Open. 2016;6(4):e010433.
Neuropsychiatric Disease and Treatment

\section{Publish your work in this journal}

Neuropsychiatric Disease and Treatment is an international, peerreviewed journal of clinical therapeutics and pharmacology focusing on concise rapid reporting of clinical or pre-clinical studies on a range of neuropsychiatric and neurological disorders. This journa is indexed on PubMed Central, the 'PsycINFO' database and CAS,

\section{Dovepress}

and is the official journal of The International Neuropsychiatric Association (INA). The manuscript management system is completely online and includes a very quick and fair peer-review system, which is all easy to use. Visit http://www.dovepress.com/testimonials.php to read real quotes from published authors. 